Iorung: : NHH SERVuCES

\title{
Assurance responsabilité civile professionnelle
} \begin{abstract}
tions adaptées à des conditions particulières. Ceci également pour l'assurance responsabilité civile professionnelle. Disposez-vous déjà d'une couverture de risque de 10 ans ou d'une somme d'assurance laquelle peut être mise à disposition deux fois par année en cas de coups durs ? Vérifiez par vous-même notre proposition et commandez une offre comparative sans engagement au moyen du coupon réponse ci-dessous.
\end{abstract}

Au nom du corps médical, nous surveillons constamment le marché des assurances et négocions pour vous des solu-

\section{ASSURANCE RESPONSABILITÉ CIVILE PROFESSIONNELLE}

\section{IF FMHSERVICES}

$\square \quad$ J'aimerais recevoir une offre de FMH Services (Insurance) pour une assurance responsabilité civile professionnelle sans frais ni engagement. (Merci de joindre une copie de votre police d'assurance actuelle.)

Domaine de spécialité

Taux d'activité

Spécialités

Pour autant que vous employez du personnel médical (sans assistantes et apprentis):

Nombre de personnes

Taux d'activité par personne

Domaine de spécialité

$\square \quad$ Veuillez m'envoyer la brochure «Responsabilité médicale: l'essentiel».

$\square \quad$ Veuillez $\mathbf{m}$ 'appeler pour un conseil personnalisé.

Prénom / Nom

Adresse

NPA / Lieu

Téléphone privé / cabinet

Atteignable le plus facilement

Adresse e-mail

\section{INSURANCE}

\section{Roth Gygax \& Partner AG}

Moosstrasse 2, 3073 Gümligen

Tél. 0319595000 - Fax 0319595010

mail@fmhinsurance.ch - www.fmhinsurance.ch

Roth Gygax \& Partner AG est une entreprise de conseil indépendante sur le plan juridique et économique recommandée par la société coopérative FMH Services. 